International Journal of Applied Mathematics

Volume 28 No. $5 \quad 2015,557-565$

ISSN: 1311-1728 (printed version); ISSN: 1314-8060 (on-line version)

doi: http://dx.doi.org/10.12732/ijam.v28i5.8

\title{
A NOTE ON THE EXISTENCE OF SOLITARY WAVES TO THE REGULARIZED BENJAMIN-ONO ZAKHAROV-KUZNETSOV (rBO-ZK) EQUATION
}

\author{
Fabián Sánchez Salazar ${ }^{1 \S}$, Miguel Angel Pachón Higuera ${ }^{2}$ \\ ${ }^{1,2}$ Department of Mathematics \\ Universidad Central
}

Kra. 5 No. 21-38 Bogotá, D.C., COLOMBIA

\begin{abstract}
In this paper, we examine the existence of solitary waves to the following equation

$$
u_{t}+a\left(u^{n}\right)_{x}+\left(b \mathscr{H} u_{t}+u_{y y}\right)_{x}=0
$$

where $\mathscr{H}$ is the Hilbert transform with respect to $x$, and $a$ and $b$ are real numbers, with $b>0$, via a variant of the mountain pass lemma.

AMS Subject Classification: 35A01, 35G25, 42B35, 35C08

Key Words: regularized Benjamin-Ono Zakharov-Kuznetsov equation, solitary waves, mountain pass lemma
\end{abstract}

\section{Introduction}

In this paper we shall present an alternative proof to that presented in [4] of the existence of solitary waves solutions to the following equation

$$
u_{t}+a\left(u^{n}\right)_{x}+\left(b \mathscr{H} u_{t}+u_{y y}\right)_{x}=0,
$$

where $\mathscr{H}$ is the Hilbert transform with respect to $x$, defined by

Received: July 4, 2015

(c) 2015 Academic Publications

${ }^{\S}$ Correspondence author 


$$
\mathscr{H}(f)(x, y)=\mathrm{p} \cdot \mathrm{v} \cdot \frac{1}{\pi} \int_{-\infty}^{\infty} \frac{f(\xi, y)}{x-\xi} d \xi
$$

when $f \in \mathcal{S}$, and $a$ and $b$ are real numbers, with $b>0$.

This equation is a bidimensional version of the regularized Benjamin-Ono equation

$$
u_{t}+a\left(u^{n}\right)_{x}+b \mathscr{H} u_{x t}=0 .
$$

For equation (1.1) has been shown the local well-posedness in Sobolev spaces and the local well and ill-posedness in weighted Sobolev spaces, also it has been proved a property of unique continuation that implies the no persistence of solutions of this in spaces of functions with arbitrary decay polynomial (see [3]). In [5] it is proved that when considering Sobolev spaces with negative indices, the map data-solution for the equation (1.1) flow is not $C_{2}$ and therefore Picard's iteration fails for those rough Sobolev spaces. Also, there is proved a global well-posedness result to this equation for small data and an interesting scattering property of these global solutions.

\section{Preliminaries}

The proof of the existence of solitary waves solutions to (1.1) presented here uses a variant of mountain pass lemma. In this section we provide some preliminary results that we shall use later. Let us recall two important lemmas whose proofs can be found in [1].

Lemma 2.1. Let $s \in(0, n / 2)$ and $f \in H^{s}\left(\mathbb{R}^{n}\right)$. Then, for $p$ such that $s=n\left(\frac{1}{2}-\frac{1}{p}\right), f \in L^{p}\left(\mathbb{R}^{n}\right)$ and

$$
\|f\|_{L^{p}} \leq c_{n, s}\left\|D^{s} f\right\|_{L^{2}} \leq c_{n, s}\|f\|_{s}
$$

where $D^{s} f=(-\Delta)^{\frac{s}{2}}=\left(|\xi|^{s} \widehat{f}\right)^{\vee}$.

Lemma 2.2. Let $s_{1}$ and $s_{2}$ be real numbers such that $s_{1}<s_{2}$. Suppose $f$ is a tempered distribution such that $D^{s_{1}} f \in L^{2}$ and $D^{s_{2}} f \in L^{2}$. Then, for $s \in\left[s_{1}, s_{2}\right], D^{s} f \in L^{2}$ and

$$
\left\|D^{s} f\right\|_{L^{2}} \leq C_{s}\left\|D^{s_{1}} f\right\|_{L^{2}}^{\theta}\left\|D^{s_{2}} f\right\|_{L^{2}}^{1-\theta}
$$

where

$$
\theta=\frac{s_{2}-s}{s_{2}-s_{1}}
$$


Lemma 2.3. If $f \in H^{1}(\mathbb{R})$, then

$$
\sup _{x \in \mathbb{R}}|f(x)| \leq\|f\|_{L^{2}(\mathbb{R})}^{1 / 2}\left\|f_{x}\right\|_{L^{2}(\mathbb{R})}^{1 / 2} .
$$

Proof. Let $f \in H^{1}(\mathbb{R})$, by the fundamental theorem of calculus and the Cauchy Schwartz inequality, we have

$$
f^{2}(x)=\int_{-\infty}^{x} 2 f(z) f_{x}(z) d z \leq C\|f\|_{L^{2}}\left\|f_{x}\right\|_{L^{2}},
$$

hence

$$
\sup _{x \in \mathbb{R}}|f(x)| \leq\|f\|_{L^{2}(\mathbb{R})}^{1 / 2}\left\|f_{x}\right\|_{L^{2}(\mathbb{R})}^{1 / 2}
$$

Definition 2.1. Let

$$
\mathscr{X}=\mathscr{X}\left(\mathbb{R}^{2}\right)=\left\{f \in L^{2}\left(\mathbb{R}^{2}\right) \mid D_{x}^{1 / 2} f \in L^{2}\left(\mathbb{R}^{2}\right) \quad \text { and } \quad \partial_{y} f \in L^{2}\left(\mathbb{R}^{2}\right)\right\}
$$

be the normed space with the norm defined by

$$
\|f\|_{\mathscr{X}}^{2}=\|f\|_{L^{2}\left(\mathbb{R}^{2}\right)}^{2}+\left\|D_{x}^{1 / 2} f\right\|_{L^{2}\left(\mathbb{R}^{2}\right)}^{2}+\left\|\partial_{y} f\right\|_{L^{2}\left(\mathbb{R}^{2}\right)}^{2} .
$$

It is clear that $\mathscr{X}$ is a Hilbert space with this norm.

As consequence of these three lemmas we have the following embedding lemma.

Proposition 2.1. For $0 \leq p \leq 4$, there exists a constant $C$, that only depend the $p$, such that, for all $f \in \mathscr{X}$,

$$
\|f\|_{L^{p+2}}^{p+2} \leq C\|f\|_{L^{2}}^{\frac{4-p}{2}}\left\|D_{x}^{1 / 2} f\right\|_{L^{2}}^{p}\left\|\partial_{y} f\right\|_{L^{2}}^{\frac{p}{2}} .
$$

In particular, if $f \in \mathscr{X}$

$$
\|f\|_{L^{p+2}} \leq C\|f\|_{\mathscr{X}}
$$

Proof. First suppose that $p<4$. By Lemma 2.3, the $\mathrm{H}$ older inequality and the Minkowski integral inequality, we have that

$$
\begin{aligned}
\int_{\mathbb{R}^{2}}|f(x, y)|^{p+2} d x d y & \leq \int_{-\infty}^{\infty} \sup _{y \in \mathbb{R}}|f(x, y)|^{p} \int_{-\infty}^{\infty} f(x, y)^{2} d y d x \\
& \leq C \int_{-\infty}^{\infty}\|f(x, \cdot)\|_{L^{2}(\mathbb{R})}^{p / 2}\left\|\partial_{y} f(x, \cdot)\right\|_{L^{2}(\mathbb{R})}^{p / 2}\|f(x, \cdot)\|_{L^{2}(\mathbb{R})}^{2} d x
\end{aligned}
$$




$$
\begin{aligned}
& \leq C \int_{-\infty}^{\infty}\left(\int_{-\infty}^{\infty} f(x, y)^{2} d y\right)^{\frac{p+4}{4}}\left(\int_{-\infty}^{\infty}\left(\partial_{y} f(x, y)\right)^{2} d y\right)^{\frac{p}{4}} d x \\
& \leq C\left\|\partial_{y} f\right\|_{L^{2}}^{\frac{p}{2}}\left[\int_{-\infty}^{\infty}\left(\int_{-\infty}^{\infty} f(x, y)^{2} d y\right)^{\frac{p+4}{4-p}} d x\right]^{\frac{4-p}{4}} \\
& \leq C\left\|\partial_{y} f\right\|_{L^{2}}^{\frac{p}{2}}\left[\int_{-\infty}^{\infty}\|f(\cdot, y)\|_{L^{\frac{2(p+4)}{4-p}}}^{2} d y\right]^{\frac{p+4}{4}} .
\end{aligned}
$$

Now, by Lemma 2.1,

$$
\|f(\cdot, y)\|_{L^{\frac{2(p+4)}{4-p}}}^{2} \leq C\left\|D_{x}^{\frac{p}{p+4}} f(\cdot, y)\right\|_{L^{2}}^{2} .
$$

On the other hand, Lemma 2.2,

$$
\left\|D_{x}^{\frac{p}{p+4}} f(\cdot, y)\right\|_{L^{2}(\mathbb{R})}^{2} \leq C\|f(\cdot, y)\|_{L^{2}(\mathbb{R})}^{\frac{2(4-p)}{p+4}}\left\|D_{x}^{1 / 2} f(\cdot, y)\right\|_{L^{2}(\mathbb{R})}^{\frac{4 p}{p+4}} .
$$

Then, the (2.3), (2.4), (2.5) and the H older inequality, we have

$$
\|f\|_{L^{p+2}}^{p+2} \leq C\left\|\partial_{y} f\right\|_{L^{2}}^{\frac{p}{2}}\|f\|_{L^{2}}^{\frac{4-p}{2}}\left\|D_{x}^{1 / 2} f\right\|_{L^{2}}^{p}
$$

Now, we show the case $p=4$. By Lemma 2.1, for all $u \in H^{1}(\mathbb{R})$ we have that

$$
\|u\|_{L^{6}} \leq C\left\|D^{\frac{1}{12}} u\right\|_{L^{4}} \leq\|u\|_{L^{4}}^{\frac{2}{3}}\left\|D^{\frac{1}{4}} u\right\|_{L^{4}}^{\frac{1}{3}} \leq\|u\|_{L^{4}}^{\frac{2}{3}}\left\|D^{\frac{1}{2}} u\right\|_{L^{2}}^{\frac{1}{3}},
$$

then, for all $f \in \mathcal{S}\left(\mathbb{R}^{2}\right)$,

$$
\int_{\mathbb{R}^{2}} f^{6}(x, y) d x d y \leq C \int_{-\infty}^{\infty}\left(\int_{-\infty}^{\infty} f^{4}(x, y) d x\right)\left(\int_{-\infty}^{\infty}\left(D_{x}^{1 / 2} f\right)^{2}(x, y) d x\right) d y
$$

On the other hand,

$$
\begin{aligned}
f^{4}(x, y) & =4 \int_{-\infty}^{y} f^{3}(x, \tilde{y}) f_{y}(x, \tilde{y}) d \tilde{y} \\
& \leq 4\left(\int_{-\infty}^{\infty} f^{6}(x, \tilde{y}) d \tilde{y}\right)^{1 / 2}\left(\int_{-\infty}^{\infty} f_{y}^{2}(x, \tilde{y}) d \tilde{y}\right)^{1 / 2}
\end{aligned}
$$

we have

$$
\int_{-\infty}^{\infty} f^{4}(x, y) d x \leq 4\left(\int_{\mathbb{R}^{2}} f^{6}(x, \tilde{y}) d x d \tilde{y}\right)^{1 / 2}\left(\int_{\mathbb{R}^{2}} f_{y}^{2}(x, \tilde{y}) d x d \tilde{y}\right)^{1 / 2} .
$$


so,

$$
\int_{\mathbb{R}^{2}} f^{6}(x, y) d x d y \leq C\left(\int_{\mathbb{R}^{2}} f^{6}(x, y) d x d y\right)^{1 / 2}\left\|D_{x}^{1 / 2} f\right\|^{2}\left\|f_{y}\right\|,
$$

it follows that

$$
\int_{\mathbb{R}^{2}} f^{6}(x, y) d x d y \leq C\left\|D_{x}^{1 / 2} f\right\|^{4}\left\|f_{y}\right\|^{2} .
$$

This shows this proposition.

The following two lemmas are similar to Lemmas 2.11 and 2.12 in [2] and their proofs follow the same ideas.

Lemma 2.4. For $0 \leq p<4$ the embedding $\mathscr{X} \hookrightarrow L_{l o c}^{p}\left(\mathbb{R}^{2}\right)$ is compact. In other words, if $\left\{\phi_{n}\right\}$ is a bounded sequence in $\mathscr{X}$ and $R>0$, there exists a subsequence $\left\{u_{n_{k}}\right\}$ of $\left\{u_{n}\right\}$ which converges strongly to $u$ in $L^{p}\left(B_{R}\right)$.

Lemma 2.5. If $\left\{u_{n}\right\}$ is bounded in $\mathscr{X}$ and

$$
\lim _{n \rightarrow \infty} \sup _{(x, y) \in \mathbb{R}^{2}} \int_{B(x, y, R)}\left|u_{n}\right|^{2} d x d y=0 .
$$

then $u_{n} \rightarrow 0$ in $L^{p}\left(\mathbb{R}^{2}\right)$.

Definition 2.2. Suppose $E$ is a real Banach space and $I \in C^{1}(E, \mathbb{R}) . I$ satisfies the Palais-Smale condition at level $c$, if there exists a sequence $\left\{u_{m}\right\}$ in $E$, such that $I\left(u_{m}\right) \rightarrow c$ and $\lim _{m \rightarrow \infty} I^{\prime}\left(u_{m}\right)=0$.

Lemma 2.6. Suppose $E$ is a Banach space and $I \in C^{1}(E, \mathbb{R})$ satisfies the following properties:

1. $I(0)=0$, and there exist $\rho>0$ and $\alpha>0$ such that $\left.I\right|_{\partial B_{\rho}(0)} \geq \alpha>0$.

2. There exist $\beta \in E-\overline{B_{\rho}(0)}$ such that $I(\beta) \leq 0$.

Let $\Gamma$ be the set all paths which connects 0 and $\beta$, i.e.,

$$
\Gamma=\{g \in C([0,1], E) \mid g(0)=0, g(1)=\beta\}
$$

and

$$
c=\inf _{g \in \Gamma} \max _{t \in[0,1]} I(g(t)) .
$$

Then $c \geq \alpha$ and $I$ possesses a Palais-Smale sequence at level $c$. 
Proof. See Theorem 2.8 in [6].

\section{Existence of Solitary Waves}

If $\phi(x-c t, y)$ is a solitary wave solution to (1.1), then

$$
-c \phi_{x}+a\left(\phi^{n}\right)_{x}+\left(-c b \mathcal{H} \phi_{x}+\phi_{y y}\right)_{x}=0 .
$$

If $\phi \in \mathscr{X}$, we can write (3.1) as

$$
-c \phi+a \phi_{n}-c b \mathcal{H} \phi_{x}+\phi_{y y}=0 .
$$

Then $\phi$ is a critical point of the functional $I$ on $\mathscr{X}$ defined as

$$
I(\phi)=\int_{\mathbb{R}^{2}} \frac{1}{2}\left[c \phi^{2}-a \frac{\phi^{n+1}}{n+1}+c b\left(D_{x}^{1 / 2} \phi\right)^{2}+\left(\partial_{y} \phi\right)^{2}\right] d x d y
$$

Therefore, in order to ensure the existence of solitary waves solutions to the equation (1.1) it is enough prove that $I$ have non-zero critical points in $\mathscr{X}$.

Let us see that $I$ satisfies the conditions or Lemma 2.6. It is obvious that $I$ is a $C^{1}$ functional for $0<p \leq 4$ and $I(0)=0$. Let $\psi \in \mathscr{X}$ be such that $\|\psi\|_{\mathscr{X}}=1$. Then for $\alpha \in \mathbb{R}$ we have

$$
\begin{aligned}
I(\alpha \psi)= & \int_{\mathbb{R}^{2}} \frac{1}{2}\left[c(\alpha \phi)^{2}-a \frac{(\alpha \psi)^{n+1}}{n+1}+c b\left(D_{x}^{1 / 2}(\alpha \psi)\right)^{2}+\left(\partial_{y}(\alpha \psi)\right)^{2}\right] d x d y \\
\geq & \frac{\min \{c, c b, 1\}}{2} \alpha^{2}\left[\int_{\mathbb{R}^{2}} \psi^{2}+\left(D_{x}^{1 / 2} \psi\right)^{2}+\left(\partial_{y} \psi\right)^{2} d x d y\right] \\
& -a \frac{\alpha^{n+1}}{n+1} \int_{\mathbb{R}^{2}} \psi^{n+1} d x d y \\
\geq & \frac{\min \{c, c b, 1\}}{2} \alpha^{2}\|\psi\|_{X}^{2}-a \frac{\alpha^{n+1}}{n+1}\|\psi\|_{L^{p+1}\left(\mathbb{R}^{2}\right)}^{p+1} \\
\geq & \frac{\min \{c, c b, 1\}}{2} \alpha^{2}-a \frac{\alpha^{n+1}}{n+1} \\
= & \alpha^{2}\left[\frac{\min \{c, c b, 1\}}{2}-a \frac{\alpha^{n-1}}{n+1}\right] .
\end{aligned}
$$

Then, taking $\alpha^{\prime}$ small enough, for instance

$$
\alpha^{\prime}<\sqrt[n+1]{\frac{(n+1) \min \{c, c b, 1\}}{2 a}},
$$


we have that $\left.I\right|_{\partial B_{\alpha^{\prime}}(0)} \geq \rho>0$, where $\rho=\left[\frac{\min \{c, c b, 1\}}{2} \alpha^{2}-a \frac{\alpha^{n+1}}{n+1}\right]$.

Let $\psi \in \mathscr{X}$ fixed such that $\|\psi\|_{\mathscr{X}}=1 \mathrm{y}\|\psi\|_{L^{p+1}}^{p+1}=c$, we have that

$$
I(\alpha \psi)=\frac{1}{2} K \alpha^{2}-\frac{1}{n+1} L \alpha^{n+1} .
$$

Taking $\alpha$ small enough, we have, $I(\alpha \psi)<0$. Also $\alpha$ can be taken large enough such that $e:=\alpha \psi \in E-\overline{B_{\alpha^{\prime}}(0)}$. This prove the second condition of Lemma 2.6. So, we have shown the following lemma.

Lemma 3.1. Let $I, \alpha$ y $\beta$ be defined as above and let $\Gamma$ and $c$ be defined as in Lemma 2.6. Then, there exists a sequence $\left\{\phi_{n}\right\}$, such that $I\left(\phi_{n}\right) \rightarrow c$ and $I^{\prime}\left(\phi_{n}\right) \rightarrow 0$.

Now, we can prove the following theorem.

Theorem 3.1. The equation (3.1) has nontrivial solutions in $\mathscr{X}$.

Proof. It is enough to show that $I$ have non-zero critical points in $\mathscr{X}$. By Lemma 3.1, there exists a Palais-Smale sequence $\left\{\phi_{n}\right\}$ at level $c$ of $I$. Therefore,

$$
\begin{aligned}
c+o(1)\left\|\phi_{n}\right\|_{\mathscr{X}} & \geq I\left(\phi_{n}\right)-\frac{\left(I^{\prime}\left(\phi_{n}\right), \phi_{n}\right) \mathscr{X}}{p+1} \\
& =\int_{\mathbb{R}^{2}} \frac{1}{2}\left(c \phi_{n}^{2}+b c\left(D_{x}^{1 / 2} \phi_{n}\right)^{2}+\left(\partial_{y} \phi_{n}\right)^{2}\right)-a \frac{\phi_{n}^{p+1}}{p+1} d x d y- \\
& -\frac{1}{p+1} \int_{\mathbb{R}^{2}}\left(c \phi_{n}^{2}+c b\left(D_{x}^{1 / 2} \phi_{n}\right)^{2}+\left(\partial_{y} \phi_{n}\right)^{2}\right)-a \phi_{n}^{p+1} d x d y \\
& \geq\left(\frac{1}{2}-\frac{1}{p+1}\right) \min \{c, c b, 1\}\left\|\phi_{n}\right\|_{\mathscr{X}}^{2},
\end{aligned}
$$

for $n$ big enough. Hence $\left\{\phi_{n}\right\}$ is bounded in $\mathscr{X}$. Considering that

$$
\begin{aligned}
0<c & =\lim _{n \rightarrow \infty} I\left(\phi_{n}\right)-\frac{1}{2}\left(I^{\prime}\left(\phi_{n}\right), \phi_{n}\right) \mathscr{X} \\
& =\lim _{n \rightarrow \infty}\left(\int_{\mathbb{R}^{2}} \frac{1}{2}\left(c \phi_{n}^{2}+b c\left(D_{x}^{1 / 2} \phi_{n}\right)^{2}+\left(\partial_{y} \phi_{n}\right)^{2}\right)-a \frac{\phi_{n}^{p+1}}{p+1} d x d y-\right. \\
& \left.-\frac{1}{2} \int_{\mathbb{R}^{2}}\left(c \phi_{n}^{2}+c b\left(D_{x}^{1 / 2} \phi_{n}\right)^{2}+\left(\partial_{y} \phi_{n}\right)^{2}\right)-a \phi_{n}^{p+1} d x d y\right) \\
& =\lim _{n \rightarrow \infty} \frac{a(p-1)}{2(p+1)} \int_{\mathbb{R}^{2}} \phi_{n}^{p+2} d x d y .
\end{aligned}
$$


Lemma 2.5 implies that

$$
\delta=\limsup _{n \rightarrow \infty} \sup _{(x, y) \in \mathbb{R}^{2}} \int_{(x, y)+\Omega} \phi_{n}^{2} d x d y>0
$$

Then, passing to a subsequence if necessary, we can assume that there exists a sequence $\left(x_{n}, y_{n}\right) \in \mathbb{R}^{2}$ such that

$$
\int_{(x, y)+\Omega} \phi_{n}^{2} d x d y>\frac{\delta}{2}
$$

for $n$ big enough. Let $\widetilde{\phi}_{n}=\phi_{n}\left(\cdot-\left(x_{n}, y_{n}\right)\right)$. Then, again passing to a subsequence if necessary, we can assume that, for some $\phi \in \mathscr{X}, \widetilde{\phi}_{n} \longrightarrow \phi$ in $\mathscr{X}$. In view of (3.4), for $n$ large enough, and Lemma 2.4, $\phi \neq 0$. Lemma 2.4 and the continuity of the function $u \rightarrow u^{p+1}$, imply that

$$
I^{\prime}(\phi)(\omega)=\lim _{n \rightarrow \infty} I^{\prime}\left(\phi_{n}\right)(\omega)=0
$$

for all $\omega \in \mathscr{X}$. This shows this theorem.

\section{Acknowledgments}

The authors were supported by the Universidad Central.

\section{References}

[1] F. Linares and G. Ponce, Introduction to Nonlinear Dispersive Equations, Universitext (2009).

[2] G. Preciado and F.H. Soriano, On the existence and analycity of solitary waves solutions to a two-dimensional Benjamin-Ono equation, arXiv, Math Appl. 1503.04291 (2015).

[3] F. Sánchez and F.H. Soriano, On the Cauchy problem associated to a regularized Benjamin-Ono-Zakharov-Kuznetsov (rBO-ZK) type equation, Submitted (2015). 
[4] F. Sánchez and F.H. Soriano, On the existence and analycity of solitary waves solutions to regularized Benjamin-Ono-Zakharov-Kuznetsov type equation, Submitted (2015).

[5] F. Sánchez and F.H. Soriano, Some remarks of the well and ill-posedness of a regularized Benjamin-Ono-Zakharov-Kuznetsov type equation. Submitted (2015).

[6] M. Willem, Minimax theorems, Progress in Nonlinear Differential Equations and Their Applications 24 (1996). 
